\title{
Summary of the National Advisory Committee on Immunization's Updated Recommendations on Human Papillomavirus (HPV) vaccines: Nine- valent HPV vaccine and clarification of minimum intervals between doses in the HPV immunization schedule
}

\author{
Tunis $\mathrm{MC}^{1}$, Deeks $\mathrm{SL}^{2,3}$ on behalf of the National Advisory Committee on Immunization (NACl)*
}

\begin{abstract}
Background: Human papillomavirus (HPV) infections are the most common sexually transmitted infections, and in the absence of vaccination it is estimated that 75 percent of sexually active Canadians will have an HPV infection at some point in their lives. Quadrivalent (HPV4) and bivalent (HPV2) vaccines have been authorized for use in Canada since 2007 and 2010, respectively. Canada's National Advisory Committee on Immunization (NACl) has previously recommended HPV4 vaccination in males and females according to a three-dose $(0,2,6$ months) or a two-dose $(0,6$ months) immunization schedule, or HPV2 vaccination for females according to a three-dose $(0,1,6$ months) or a two-dose $(0,6$ months) immunization schedule, depending on the age and health status of the recipient. In February 2015, a nine-valent (HPV9) vaccine (Gardasil ${ }^{\otimes}$, Merck Canada, Inc.) was authorized for use in Canada for the prevention of HPV types 6-, 11-, 16-, 18-, 31-, 33-, 45-, 52- and 58-related cancers and anogenital warts (AGW) in females aged 9 to 45 years and males aged 9 to 26 years.
\end{abstract}

Objectives: To summarize evidence on the new HPV9 vaccine and make recommendations for its use in Canada, to review epidemiological data on the relative contribution to disease outcomes of the 5 additional genotypes covered in the HPV9 vaccine, and to clarify acceptable minimum intervals between vaccine doses in either a 2-dose or 3-dose HPV immunization schedule.

Methods: The NACI HPV working group performed literature reviews on the topics of HPV vaccine minimum dose intervals, and the HPV9 vaccine. Vaccine manufacturers provided additional data for review. All evidence was reviewed, rated, and a representative dataset for each trial was reported in evidence tables. A knowledge synthesis was performed, and $\mathrm{NACl}$ approved specific evidence-based recommendations, elucidating the rationale and relevant considerations.

Results: At the time of the review, only one published peer-reviewed study of HPV9 vaccine was available for inclusion, but information from additional unpublished studies in the form of presentations, posters, and abstracts were shared by the vaccine manufacturer to be appraised.

Based on the evidence available to date, the HPV9 vaccine is recommended on a three-dose schedule for females aged 9 to 26 years; females aged over 26 years who have not been vaccinated previously or who have not completed the vaccination series; and males aged 9 to 26 years. There is insufficient evidence at this time to recommend a two-dose immunization schedule with HPV9 vaccine, but a clinical trial to assess alternate dosing schedules for HPV9 vaccine is currently underway. The efficacy of HPV9 vaccine in preventing infection and disease related to HPV types $31,33,45,52$ and 58 in individuals previously immunized with HPV4 vaccine has not been assessed. In Canada, immunization against HPV types 16 and 18 with HPV2, HPV4 or HPV9 vaccine can prevent approximately $70 \%$ of anogenital cancers and $60 \%$ of high-risk precancerous cervical lesions. Immunization with either HPV4 or HPV9 vaccine can prevent approximately $90 \%$ of AGWs (HPV types 6 and 11). Immunization with HPV9 vaccine can prevent up to an additional $14 \%$ of anogenital cancers and up to $30 \%$ of high-risk precancerous cervical lesions caused by the additional five HPV types $(31,33,45,52$ and 58 ) against which the vaccine protects. The disease burden associated with the five additional genotypes contained in HPV9 vaccine is not equally shared between the sexes, with the additional benefit primarily observed among females.

\section{Affiliations}

${ }^{1}$ Centre for Immunization and Respiratory Infectious Diseases, Public Health Agency of Canada, Ottawa, ON

${ }^{2} \mathrm{NACl}$ HPV Working Group Chair, Toronto, ON

${ }^{3}$ Immunization and Vaccine Preventable Diseases, Public Health Ontario, Toronto, ON

*Correspondence: naci-ccni@ phac-aspc.gc.ca 
In terms of the HPV immunization schedule, there is a paucity of published evidence supporting shortened or flexible minimum intervals for HPV vaccines, compared to ample evidence endorsing the recommended schedules as well as evidence supporting delays in the receipt of booster doses. Assumptions about the immunogenicity and efficacy of shortened 'flexibility range' minimum dose intervals rely heavily on the manufacturer's unpublished data on file and their Health Canada-approved recommendations included in the product monographs. The $\mathrm{NACl}$ recommendations and evidence grades based on these results are included below.

Conclusions: In addition to the HPV 6, 11, 16, and 18 strains that can be covered by other HPV vaccines, the HPV9 vaccine is expected to provide further protection by preventing infection and disease related to HPV types $31,33,45,52$ and 58 . Protecting against these additional strains may prevent up to an additional $14 \%$ of anogenital cancers and up to $30 \%$ of high-risk precancerous cervical lesions in Canada. Efforts should be made to administer HPV vaccines at the recommended intervals. When an abbreviated schedule is required, minimum intervals between HPV vaccine doses should be met including a minimum interval of 24 weeks between the first and last dose in either a 2-dose or 3-dose schedule. Please note that $\mathrm{NACl}$ is currently reviewing evidence for a 2-dose HPV9 vaccine immunization schedule.

Suggested citation: Tunis MC, Deeks SL on behalf of the National Advisory Committee on Immunization (NACl). Summary of the National Advisory Committee on Immunization's Updated Recommendations on Human Papillomavirus (HPV) vaccines: Nine-valent HPV vaccine and clarification of minimum intervals between doses in the HPV immunization schedule. Can Comm Dis Rep 2016;42:149-51. https://doi.org/10.14745/ccdr.v42i07a03

\section{Introduction}

The review of the literature on human papillomavirus (HPV) vaccines: Nine-valent HPV vaccine and the National Advisory Committee on Immunization's (NACl) current HPV vaccine recommendations are published in the full NACl statement (1) and the HPV chapter of the Canadian Immunization Guide (2).

\section{Recommendation no.1}

\section{$\mathrm{NACl}$ concludes that any of the currently authorized HPV vaccines in Canada can be used according to the recommended HPV immunization schedules - NACI recommendation evidence grade $A$ or $B$ (Table 1).}

HPV immunization may be completed with HPV2, HPV4 or HPV9 vaccines in females and HPV4 or HPV9 vaccines in males, according to the immunization schedules summarized in Table 1, below. Where possible, the same vaccine should be used to complete the series. If completion of the series with the same vaccine is not possible, the HPV2, HPV4 or HPV9 vaccine may be used to complete the series in females, and the HPV4 or HPV9 vaccine may be used to complete the series in males. The HPV9 vaccine among immunocompetent 9 to 26 years old is expected to provide similar protective efficacy against genotypes contained in the HPV4 vaccine. HPV2, HPV4 and HPV9 vaccines all protect against HPV types 16 and 18, which are responsible for approximately $70 \%$ of anogenital cancers. In addition, HPV9 vaccine protects against the additional five HPV types not contained in HPV4 vaccine (HPV 31, 33, 45, 52 and 58).

HPV9 protects against 5 additional HPV genotypes responsible for approximately $14 \%$ of anogenital cancers. HPV4 and HPV9 also protect against HPV genotypes 6 and 11, which cause over $90 \%$ of AGWs. At the population level, if all persons recommended for the vaccine receive it, and there is $100 \%$ long-term efficacy, immunization with HPV9 vaccine in Canada can potentially prevent annually up to 320 additional cases of anogenital cancers (300 in females and 20 in males). Adverse events following immunization with HPV vaccines primarily include mild to moderate injection site-related pain, erythema and swelling. These local adverse events are more common in HPV9 vaccine recipients compared to recipients of the HPV4 vaccine.

$\mathrm{NACl}$ will reassess the grading of this recommendation as new evidence emerges.

\section{Table 1: Recommended Immunization Schedule with Human Papillomavirus Vaccines}

\begin{tabular}{|c|c|c|}
\hline Recommended groups & $\begin{array}{l}\text { Recommended } \\
\text { immunization } \\
\text { schedule }\end{array}$ & $\begin{array}{l}\text { Vaccine(s) } \\
\text { and } \mathrm{NACl} \\
\text { evidence } \\
\text { grade }\end{array}$ \\
\hline \multirow{2}{*}{$\begin{array}{l}\text { Healthy (immunocompetent, } \\
\text { non-HIV infected) Females } \\
9-14 \text { years of age (and healthy } \\
\text { females }>15 \text { years of age } \\
\text { in whom the first dose was } \\
\text { administered between } 9-14 \\
\text { years of age) }\end{array}$} & $\begin{array}{l}\text { 2- or 3-dose } \\
\text { schedule }\end{array}$ & $\begin{array}{l}\text { HPV2 or HPV4 } \\
\text { (Grade A) }\end{array}$ \\
\hline & 3-dose schedule & $\begin{array}{l}\text { HPV9 } \\
\text { (Grade B) }\end{array}$ \\
\hline $\begin{array}{l}\text { Healthy (immunocompetent, } \\
\text { non-HIV infected) Females } \\
>15 \text { years of age }\end{array}$ & 3-dose schedule & $\begin{array}{l}\text { HPV2 or HPV4 } \\
\text { (Grade A) or } \\
\text { HPV9 (Grade B) }\end{array}$ \\
\hline \multirow{2}{*}{$\begin{array}{l}\text { Healthy (immunocompetent, } \\
\text { non-HIV infected) Males 9-14 } \\
\text { years of age (and healthy } \\
\text { males }>15 \text { years of age in } \\
\text { whom the first dose was } \\
\text { administered between 9-14 } \\
\text { years of age) }\end{array}$} & $\begin{array}{l}\text { 2- or 3-dose } \\
\text { schedule }\end{array}$ & HPV4 (Grade B) \\
\hline & 3-dose schedule & HPV9 (Grade B) \\
\hline $\begin{array}{l}\text { Healthy (immunocompetent, } \\
\text { non-HIV infected) Males }>15 \\
\text { years of age }\end{array}$ & 3-dose schedule & $\begin{array}{l}\text { HPV4 or HPV9 } \\
\text { (Grade B) }\end{array}$ \\
\hline $\begin{array}{l}\text { Immunocompromised } \\
\text { individuals and } \\
\text { immunocompetent } \\
\text { HIV-infected individuals }\end{array}$ & 3-dose schedule & $\begin{array}{l}\text { HPV2, HPV4 or } \\
\text { HPV9 in females; } \\
\text { HPV4 or HPV9 in } \\
\text { males } \\
\text { Grade I }\end{array}$ \\
\hline
\end{tabular}




\section{Recommendation no. 2}

$\mathrm{NACl}$ concludes that there is insufficient evidence at this time to recommend a 2-dose immunization schedule with HPV9 vaccine - NACI Recommendation Evidence Grade I.

A phase III clinical trial to study the safety and immunogenicity of a 2-dose immunization schedule with HPV9 vaccine is currently under way. The goal of the 37-month study is to establish whether the investigational 2-dose regimens of 0, 6 months and 0,12 months in boys and girls 9 to 14 years of age are safe and immunogenic, with an antibody response non-inferior to that observed in females 9 to 26 years of age who received the standard 3-dose regimen of the vaccine.

$\mathrm{NACl}$ will review and reassess this recommendation as new evidence emerges.

\section{Recommendation no. 3}

$\mathrm{NACl}$ concludes that there is insufficient evidence at this time to recommend, at a population level, the re-immunization with HPV9 vaccine of individuals who have completed an immunization series with another HPV vaccine - NACI Recommendation Evidence Grade I.

Unpublished data suggest that re-immunization with HPV9 vaccine after completion of a series with HPV4 produces lower immunogenicity to the five additional HPV genotypes (clinical significance unknown) and higher incidences of local injection site adverse events; efficacy has not been assessed.

While not recommended at a population level, individuals who have been vaccinated with HPV4 vaccine and who wish to take advantage of the additional protection provided by HPV9 vaccine may be vaccinated with HPV9 vaccine. There is insufficient evidence at this time to determine whether fewer than 3 doses of HPV9 vaccine conveys protection against the additional five HPV types in prior HPV4 vaccine recipients.

$\mathrm{NACl}$ will review and reassess this recommendation as new evidence emerges.

\section{Recommendation no. 4}

$\mathrm{NACl}$ concludes that there is good evidence that the minimum interval between the first and last doses in either a 2-dose or 3-dose HPV immunization schedule should be 24 weeks (6 months) - NACI Recommendation Evidence Grade A.

$\mathrm{NACl}$ recommends that, whenever possible, the recommended intervals between doses of HPV2 vaccine $(0,1,6$ months in a 3 -dose schedule or 0 and 6 months in a 2-dose schedule), HPV4 vaccine $(0,2,6$ months in a 3 -dose schedule or 0 and 6 or 12 months in a 2-dose schedule) and HPV9 vaccine (0,2 and 6 months) should be respected. When an abbreviated schedule is unavoidable, the minimum intervals in a 3-dose schedule (as summarized in Table 1) between the first and second doses of HPV vaccine is 4 weeks (1 month), the minimum interval between the second and third doses of HPV vaccine is 12 weeks

( 3 months), and the minimum interval between the first and third doses is 24 weeks ( 6 months). The minimum interval between the first and second dose in a 2-dose schedule with either HPV2 or HPV4 is 24 weeks (6 months).

\section{Conflict of interest}

None.

\section{Funding}

The work of $\mathrm{NACl}$ is supported by the Public Health Agency of Canada.

\section{References}

1. National Advisory Committee on Immunization's Updated Recommendations on Human Papillomavirus (HPV) Vaccines: 9-valent HPV vaccine and clarification of minimum intervals between doses in the HPV immunization schedule. http://www.healthycanadians.gc.ca/ publications/healthy-living-vie-saine/human-papillomavirus9-valent-vaccine-update-recommendation-misesa-jour-recommandations-papillome-humain-vaccin-nona valent/index-eng.php.

2. National Advisory Committee on Immunization. Canadian Immunization Guide: Part 4: Human papillomavirus vaccine. Ottawa (ON): Public Health Agency of Canada; [modified 2016 July 07]. http://www.phac-aspc.gc.ca/publicat/cig-gci/ p04-hpv-vph-eng.php. 